\title{
Téoros
}

Revue de recherche en tourisme

\section{Le tourisme d'aventure : quand le succès peut être mortel}

\section{Michel Zins}

Volume 13, numéro 3, automne 1994

Le tourisme d'aventure : vers la maturité ?

URI : https://id.erudit.org/iderudit/1077105ar

DOI : https://doi.org/10.7202/1077105ar

Aller au sommaire du numéro

Éditeur(s)

Université du Québec à Montréal

ISSN

0712-8657 (imprimé)

1923-2705 (numérique)

Découvrir la revue

Citer ce document

Zins, M. (1994). Le tourisme d'aventure : quand le succès peut être mortel. Téoros, 13(3), 2-2. https://doi.org/10.7202/1077105ar d'utilisation que vous pouvez consulter en ligne.

https://apropos.erudit.org/fr/usagers/politique-dutilisation/ 


\title{
Présentation Le tourisme d'aventure:
quand le succès peut être mortel
}

\author{
Michel Zins
}

Le tourisme d'aventure est-ilpris de vertige ou de nausée? Ou encore vit-il des angoisses métaphysiques propres a ladolescence ou la quarantaine? De toute evidence, id a connu et connait encore une croissance importante. Ila aussi gagné une certaine maturité, autant en ce qui concerne sa clientéle que les entreprises qui y operent.

L'engouement grandissant pour la nature et les experiences physiques, mythiques et sensonielles qu'elle permet de vivre offre des perspectrves de succes pour de nombreuses entreprises et régions du monde. Cet engouement porte en lui des menaces importantes pour l'existence meme du tourisme d'aventure.

Le tourisme d'aventure est rattrapé et submergéà certains egards par un tourisme plus englobant. l'écotounisme, sintéressant à la nature et aux populations locales, mais sans la notion de risque. Le tourisme actif ou sportif, où la nature n'est parfos qu'un terrain de jeu, comme le rafting, la motoneige, voire le ski, offre une aventure d'unautre type. Sans compter les concepts interactifs et dimagerie virtuelle qui donnent des frissons dans une salle ou un simulateur.

Le tourisme d'aventure est-il une dernière frontière de lhumanité en quête de vérité et d'authenticité? Est-ilune industrie qu'on peut gérer comme une autre?

Normand Cazelais questionne donc d juste titre la signification du concept mème de tourisme daventure et remet en doute son existence propre. On devrait plutot parler selon lui de tounisme de linconfort programmé, dans des environnements differents ou exotrques, ou liaventure reste somme toute trés encadreve et le risque contrólé.

Philippe Bourdeau présente quant à hi une analyse originale des motivations culturelles et exis tentielles qui sous-tendent de nos jours la demande de tourisme d'aventure, et il conclue que le tourisme d'aventure repond a un besoin ud'ailleursm et d'imaginaire et même d'envement sensoriel, que l'on ne trouve plus dans les societés urbanisées et organisées à outrance d"Europe de l'Ouest et d"Amérique du Nord.

Jean-Claude Jay-Ravon, en collaboration avec Brigute Morneau, suggèrent cependant que le tourisme d'aventure est de nos jours plus une façon de rejoindre les lieux et ewironnements de l'ancienne Aventure, qu'une véritable aventure et s'enricht de laspect culturel des heux, des etres rencontrés et des expériences vécues plutôt que simplement de laspect physique et naturel. Le Québec a tout pour offrir ce gerre d'expénence, au niveau intemational, a condition de pouvoir en synthétiser les differentes facettes dans une experience d'ensemble dans des endroits précis du terntorre qui seraient sanctwarisés vove usacralisésn.

Yves Tinard nous parle quant a lui de lexpérience francaise, ou le tounisme d"aventure est fortement médiatsé. Des émissions télévisées a forte cote d'écoute y sont consacrées telles Thalassa ou Ushuaila. Des événements comme le raid Paris-Dakar, Haricana sont très courus. Les aventuriers qui prennent des risques en mer. en montagne, ou dans le désert sont des héros. Médecins Sans Frontiere et laventure humaine sous toutes ses formes sont da la une des nouvelles. On y trouve cependant toujours l'éloignement et un cadre géagraphique exotique puissant. une composante sportive ou physique forte, des risques et aléas réels, linconfort et /imaginaire.
L'élément d'unicité et le caractère men voie de disparition sont trés valorisés.

Le risque de banalisation devient alors la menace la plus grande pour lamateur français d'aventure.

II donne des conseils pour créer et gérer un produit d'aventure dont les grands enjeux sont la saisonnahté, les ressources humaines au statut précaire, la concurrence de plus en plus vive, la réglementation et les risques à gérer.

Peter W. Whams, Kent Stewart et Bernard Campbell decrivent "evolutionet lasituation actuelle du tourisme d"aventure dans /Ouest" canadien. Bien que cette partie du Canada a donné toute sa place au tourisme d'aventure sous différentes formes, les entreprises qui y opérent restent prises avec des difficultés importantes en terme de gestion, de mise en marché et de croissance.

Jean Michauda analysé, dans divers textes, le discours promotionnel des marchands d'aventure à l'égard du trekking. qui incame le tourisme d'aventure le plus apurn. Si l'origine du terme signifie uun voyage long et difficilen, le mot est utilise comme si tout le monde comprenait ce qu'il sous-entend. Bien qu'utilisé avec plus ou moins de variances, on y associe toujours une nature difficile et la rencontre humaine.

Renée Dubéet MichelZins analysentles enieuxparticulers auxquels est confronté une entreprise ou une région voulant mettre en marche un produit de tourisme d'aventure. Devant un marché segmenté, on ne peut pas étre tout pour tout le monde. Tout devantêtre accessible. trop de monde banalise le produit. II faut aussi savoir rejoindrele client recherché et lui parler de la bonne facon.

André Hut trace l'évolution du tourisme d'aventure des privilégiés anglars, qui on tinventé le tourisme, qui était al l'époque une aventure de toute facon au début du siècle, aux premiers touristes d'aprés guerre, en passant par les hippies et routards et enfin les yuppies d'aujourd'hul à la recherche d'experiences fortes. II met en garde contre une aporoche egoiste et inconsciente, sans égardaux peuples et environnements visités, et voire violés.

Richard Bangs, de Mountain Travel Sobek, methumoristiquementen garde les professionnels de cette industrie contre les deux (2) plus grands risques : le touriste qui n'a pas sa place dans un tel vovage, par manque de réalisme, de crvilité ou d'attitude adequate, le touropérateur mal organisé ou manquant d'éthique.

Oswaldo Munoz, finalement, insiste sur la nécessité pour le tounisme d'aventure d'être écotouristique. II met en lumière la valeur économique et sociale réelle d'un tourisme pratiqué dans la nature de façon respectueuse, L'écotourisme aide ainsi, sill est bien compris, à justifier économiquement la conservation des espaces encore vierges qui existent sur cette planete.

La gestion du tourisme d'aventure représente peut-être une des dernieres aventures waiment humaine. II taut reussir car elle porte en elle la jus tification économique de la préservation de lá nature et des espaces qui sont des vestiges et des souvenirs des aventures passees de thumanite.

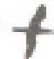

